\title{
Floral scent composition predicts bee pollination system in five butterfly bush (Buddleja, Scrophulariaceae) species
}

\author{
W.-C. Gong ${ }^{1,2, \dagger}$, G. Chen ${ }^{1, \dagger}$, N. J. Vereecken ${ }^{3}$, B. L. Dunn ${ }^{4}$, Y.-P. Ma ${ }^{1}$ \& W.-B. Sun ${ }^{1}$ \\ 1 Kunming Botanical Garden, Kunming Institute of Botany, Chinese Academy of Sciences, Kunming, Yunnan, China \\ 2 University of Chinese Academy of Sciences, Beijing, China \\ 3 Landscape Ecology \& Plant Production Systems, Interfaculty School of Bioengineering (EIB), Université Libre Bruxelles (ULB), Brussels, Belgium \\ 4 Department of Horticulture and Landscape Architecture, Oklahoma State University, Stillwater, MN, USA
}

\section{Keywords}

Bee pollination; breeding system; butterfly bush; floral scent; plant-pollinator interaction; Sino-Himalayan region; species-specific.

\section{Correspondence}

W.-B. Sun, Kunming Botanical Garden,

Kunming Institute of Botany, Chinese

Academy of Sciences, Kunming, 650201

Yunnan, China.

E-mail:wbsun@mail.kib.ac.cn

'These authors contributed equally to this work.

Editor

A. Dafni

Received: 31 October 2013; Accepted: 31 January 2014

doi:10.1111/plb.12176

\begin{abstract}
Traditionally, plant-pollinator interactions have been interpreted as pollination syndrome. However, the validity of pollination syndrome has been widely doubted in modern studies of pollination ecology. The pollination ecology of five Asian Buddleja species, B. asiatica, B. crispa, B. forrestii, B. macrostachya and B. myriantha, in the Sino-Himalayan region in Asia, flowering in different local seasons, with scented inflorescences were investigated during 2011 and 2012. These five species exhibited diverse floral traits, with narrow and long corolla tubes and concealed nectar. According to their floral morphology, larger bees and Lepidoptera were expected to be the major pollinators. However, field observations showed that only larger bees (honeybee/bumblebee) were the primary pollinators, ranging from $77.95 \%$ to $97.90 \%$ of total visits. In this study, floral scents of each species were also analysed using coupled gas chromatography and mass spectrometry (GC-MS). Although the five Buddleja species emitted differentiated floral scent compositions, our results showed that floral scents of the five species are dominated by substances that can serve as attractive signals to bees, including species-specific scent compounds and principal compounds with larger relative amounts. This suggests that floral scent compositions are closely associated with the principal pollinator assemblages in these five species. Therefore, we conclude that floral scent compositions rather than floral morphology traits should be used to interpret plant-pollinator interactions in these Asian Buddleja species.
\end{abstract}

\section{INTRODUCTION}

Plant-pollinator interactions have been a central topic for evolutionary biologists since the time of Darwin, as a model system for the study of adaptation and evolution. Traditionally, plant-pollinator interactions are usually interpreted using the concept of the pollination syndrome, since Fredrico Delpino first put forward this concept (Delpino 1873-1874; Faegri \& Van der Pijl 1979; Fenster et al. 2004). Pollination syndromes are suites of floral traits, such as shape, colour and odour that were hypothesised to reflect convergent adaptations of flowers for pollination by specific types of animal (Van der Pijl 1961; Faegri \& Van der Pijl 1979; Fenster et al. 2004). The predictive power of the pollination syndrome has been widely accepted in describing specialised plant-pollinator relationships (Darwin 1862; Vogel 1954; Faegri \& Van der Pijl 1979; Fenster et al. 2004). However, modern studies of pollination ecology have emphasised the tendency for ecological generalisation in pollination system or diverse animal visitors of plant taxa (Waser et al. 1996; Ollerton et al. 2006, 2009). Furthermore, there is not always a clear boundary between different pollination syndromes (Ollerton \& Watts 2000), so it might be difficult to attribute a flower to a specific pollination syndrome. Thus, the validity of pollination syndromes in modern pollination ecology does not always apply.
There are alternative approaches to interpret plant-pollinator interactions, with emphasis on different underlying factors. For example, Ellis \& Ellis-Adam (1993) provided a typology, which is based on Müller's (1881) functional classification of flower types, interpreting modern plant-pollinator relationships. In this typology, the correlations between floral morphology traits and insects, especially relative length of the corolla tube and tongue of the insect pollinator, nectar volume per flower and diet of insects were considered significant (Ellis \& Ellis-Adam 1993; Waser \& Ollerton 2006). Generally, pollinators not only respond to visual cues of plant species (Galen 1989; Fenster et al. 2004; Pauw 2006; Parachnowitsch \& Kessler 2010; Kulbaba \& Worle 2012), but also to olfactory cues, such as odour (Raguso 2001, 2008; Schiestl 2010). Although odour features (e.g., presence versus absence, and how flowers smell) were considered an important component of traditional the pollination syndrome, the roles of floral scent compositions in chemical communications with pollinators have been highlighted in modern pollination ecology (Raguso 2008; Shuttleworth \& Johnson 2009; Schiestl 2010; Schiestl \& Dötterl 2012). Furthermore, floral scent compositions could potentially mediate plant-pollinator interactions, and promote specialisation in pollination systems, notably through specific floral scent compounds in scented flowers (Raguso 2008; Shuttleworth \& Johnson 2009; Soler et al. 2010). 
Traditionally, Buddleja L. (Scrophulariaceae) species are inferred as pollinated by butterflies, and therefore commonly known as a 'Butterfly Bush' (Faegri \& Van der Pijl 1979; Proctor et al. 1996; Norman 2000). The common name, 'Butterfly Bush', arose soon after the cultivation of $B$. davidii, which does attract butterflies (Faegri \& Van der Pijl 1979; Andersson et al. 2002; Chen et al. 2011). Sweet scent, massed flowers (presumably as a platform) and slender corolla tube with reverse herkogamy are all considered to be associated with butterfly pollination (Webb \& Lloyd 1986; Andersson \& Dobson 2003; Willmer 2011). Here, we described plant-pollinator interactions of five Asian Buddleja species (Buddleja asiatica Lour., B. crispa Benth., B. forrestii Diels, B. macrostachya Benth., B. myriantha Diels), whose relationships between plants and pollinators have never been explored. Plant-pollinator interactions in these Buddleja species cannot be interpreted through a pollination syndrome. According to the typology of Ellis \& Ellis-Adam (1993), these five Buddleja species should mainly be pollinated by larger bees (including Apidae) and Lepidoptera because of their long floral tubes (Waser 2006). Buddleja davidii Franch. is a popular butterfly bush, not only because of the attraction to butterflies, but also the abundant emission of 4-oxoisophorone (Andersson \& Dobson 2003; Guédot et al. 2008). Studies on floral scent-pollinator relationships of $B$. davidii showed that floral scent composition is an important attractant for the major pollinators (Andersson et al. 2002; Andersson \& Dobson 2003; Guédot et al. 2008). Such studies further evoke our interest in exploring the interactions between floral scent composition and principal pollinators in Buddleja species.

In this study, our objectives were (i) to interpret plant-pollinator interactions in these five Buddleja species according to the topology of Ellis \& Ellis-Adam; (ii) to record the primary pollinator assemblage of the five Buddleja species; and (iii) to explain the inconsistency between expected pollinator assemblages and field observations through floral scent composition, and explore plant-pollinator interactions among these five species. Floral scent is a complex phenotypic trait, with diverse chemical compositions and relative amounts, and each factor may affect the relationships between floral scent and pollinator. Combined with the chemical ecology functions of floral scents in other angiosperms, plant-pollinator interactions in the five Buddleja species are further interpreted.

\section{MATERIAL AND METHODS}

Study species and sites

The study was carried out from January to August in 2011 and 2012, in the Sino-Himalayan region of Sichuan and Yunnan Provinces, China. This area is part of a global biodiversity hotspot, and the secondary centre of origin for Asian Buddleja species that originate from South Africa, with over 90\% of the Asian Buddleja species being distributed there (Moore 1947; Li \& Leeuwenberg 1996; Nie et al. 2005; Chen et al. 2007). Five hermaphroditic and perennial shrubs, B. asiatica, B. crispa, $B$. forrestii, B. macrostachya and B. myriantha, were studied from the region (Fig. 1). Buddleja asiatica is widely distributed in Asia, while the other four species are mainly distributed in the Sino-Himalayan region. The five species mainly grow in disturbed areas along roadsides, and flower in different local seasons, with scented inflorescences. All studies described
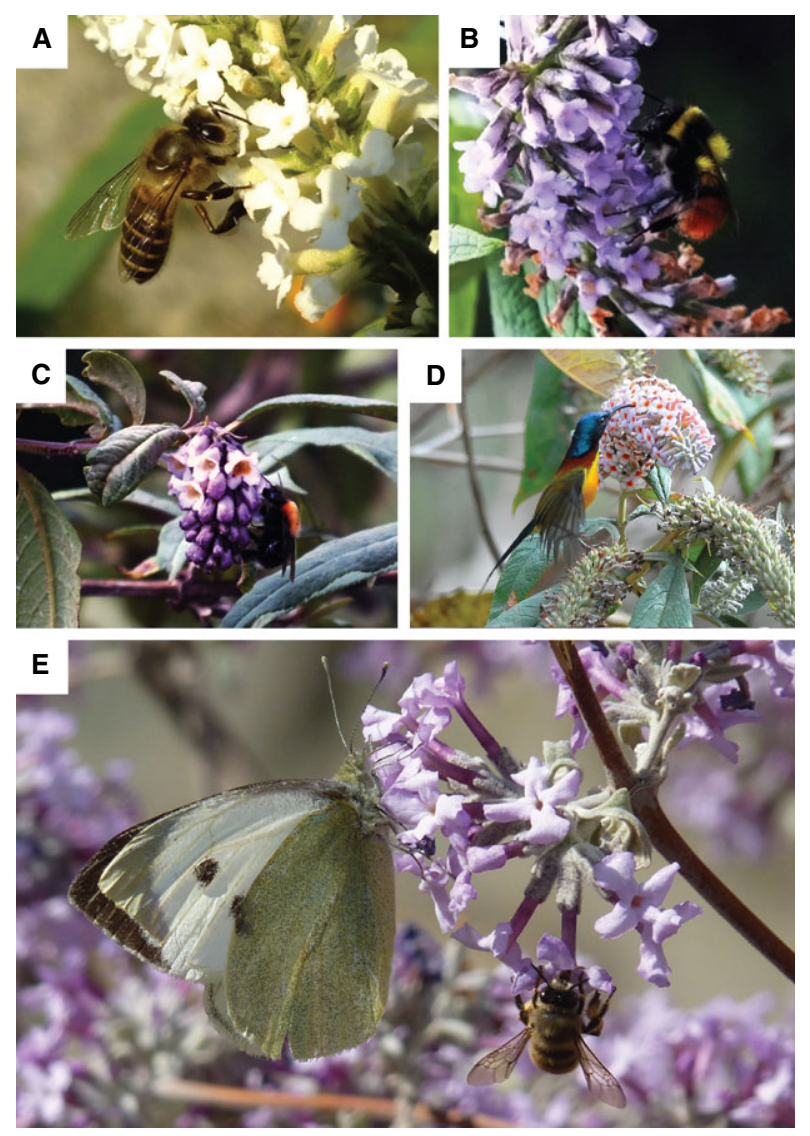

Fig. 1. Plant-pollinator interactions of five Buddleja species investigated in the Sino-Himalayan region: (A) B. asiatica (B) B. myriantha (C) B. forrestii (D) B. macrostachya (E) B. crispa. Buddleja macrostachya was visited by the sunbird Aethopyga nipalensis (Passeriformes, Nectariniidae); B. crispa was visited by Pieris sp. (Lep. Pieridae) and Apis cerana (Hym. Apidae). Photographs by W.-C. Gong (A, B, C, E) and X. Yuan (D).

herein were performed in natural populations. Detailed information is listed in Table S1. Vouchers specimens of each species were deposited at the Herbarium of the Kunming Institute of Botany (KUN), the Chinese Academy of Sciences.

\section{Observation of visitor assemblages}

Inflorescence visitor observations were conducted at peak flowering times during 2011 and 2012. Initially, we patrolled each selected population and gathered information of all possible visitors from $08: 30$ to 22:00 h for 1 day following the methods of Huang \& Fenster (2007). Subsequently, diurnal visitors were recorded at 08:30-09:00, 10:00-10:30, 12:00-12:30, 14:00$14: 30,16: 00-16: 30$ and 18:00-18:30 h. Floral visitors of $B$. asiatica were recorded on sunny and warm days (12 days) between 19 February-9 March 2011, 25-28 February 2012; B. crispa was observed between 23-25 March 2011, 23-27 February 2011 (7 days); B. myriantha was observed between 7 June-11 July 2011 (6 days); B. forrestii was observed between 19-24 June 2011 (3 days); B. macrostachya was observed between 5-9 August 2011, 14-19 August 2012 (6 days). In addition, nocturnal visitors (between 20:00-22:00 h) of each plant species were also observed after daytime observations. It should be noted that when it was raining, field observation 
were stopped. The studied populations of each species are listed in Table S1.

In each study population, three to five individual plants (at least $5 \mathrm{~m}$ apart) with similar inflorescence sizes and numbers were randomly selected to record the behaviours and residence time of visitors. A flower visit was defined as the period between first landing on the inflorescence and final departure. All types of insect visitors were caught and immediately inspected for pollen presence (indicating potential role as pollinator) using LED Illumination (Meilun, Shenzhen, China; LED lamps, 40 of $25 \mathrm{~mm}$ ) on separate days after visitor observations to avoid disturbance to visitor activity. Three to 10 insects per species were collected for further species identification and voucher specimens. Floral visitors were recorded by human observers and photographed with a digital camera (Fuji, Tokyo, Japan; Fujifilm FinePix HS22EXR) to allow classification into different functional groups (honeybee, bumblebee, butterfly, hawkmoth, hoverfly, fly and sunbird). Functional groups were retained instead of taxonomic species as classification units, because the same functional group of pollinators was predicted to exert similar selection on the flowers (Fenster et al. 2004; Gong \& Huang 2011).

Natural populations of Buddleja were mainly in disturbed habitats adjacent to roads, and so easily destroyed. All populations were disturbed and available in only one of the two study years for visitor observations, although a total of 11 populations were studied.

\section{Visitation frequency and pollinator diversity}

Visitation frequency (per inflorescence per hour) of each functional group was calculated for each plant species. However, it is difficult to quantify pollen deposition during a single visit from the pollinator, but the specific floral design of Buddleja, e.g., narrow corolla tube with thick trichomes inside, and reverse herkogamy (stigma below the anthers, with inserted anthers) ensured effective pollen deposition and transfer for each visit (W.-C. Gong, personal observation).

Pollinator generalisation (PG) level (Gong \& Huang 2009) was calculated as the pollinator diversity for each plant species, using Simpson's diversity index $\left(\frac{1}{D}\right)$ (Sahli \& Conner 2006):

$$
\frac{1}{\mathrm{D}}=\frac{1}{\sum_{\mathrm{i}=1}^{\mathrm{S}} \mathrm{P}_{\mathrm{i}}^{2}}
$$

where $\mathrm{p}_{\mathrm{i}}$ is the proportion of visits made by pollinator functional group $\mathrm{i}$ and $\mathrm{S}$ is the total number of functional groups visiting the species. A value of one indicates absolute specialisation on one functional group.

Floral visitor evenness $\left(\mathrm{E}_{\frac{1}{\mathrm{D}}}\right)$ of each species was calculated by dividing Simpson's diversity index by the number of functional groups of floral visitors (S) (Sahli \& Conner 2006):

$$
\mathrm{E}_{\frac{1}{\mathrm{D}}}=\frac{\frac{1}{D}}{\mathrm{~S}}
$$

\section{Measurement of floral morphology and reward}

Floral traits of the five study Buddleja species with respect to pollinator attraction, reward and pollen transfer were measured. For at least two flowers per plant, ten different plants in each population were randomly selected. Inflorescence dimensions (length and width), floral length (height) and width of the corolla tube were measured. Floral display (number of fully opened flowers per inflorescence at peak flowering time) and floral longevity (in days) of single flowers were also recorded, and the degree of reverse herkogamy (in $\mathrm{mm}$ ) was measured. This last parameter refers to the position of the stigma below the level of the anthers, a trait causing pollinators to first contact the anthers before the stigma. For this reason, reverse herkogamy is expected to facilitate higher pollen export and is typically associated with pollination by Lepidopteron species (Endress 1996). The number of pollen grains and ovules of each flower was counted, as in Wyatt et al. (2000). The stigma receptivity and pollen viability of pre-flowering and florescence stages were tested following Mao \& Huang (2009).

We also estimated nectar production for each Buddleja species. The fully developed inflorescences were individually bagged with nylon netting to exclude floral visitors before nectar measurement. Buddleja forrestii and B. macrostachya produced a larger volume of nectar, so nectar was directly absorbed using 20- $\mu$ l calibrated pipettes (Drummond Scientific Co., Broomall, PA, USA), and at least 20 single flowers were sampled, with two flowers per plant. B. asiatica, B. crispa and $B$. myriantha produced less nectar volume, so nectar was collected from 20 individual flowers of each species per time and centrifuged at $3000 \mathrm{rpm}$ for $10 \mathrm{~s}$, with three replicates. Sugar concentration of all nectar collected was also measured using a handheld refractometer (Bellingham \& Stanley, Kent, UK; Eclipse 45-81: 0-50\% sucrose, w/w).

\section{Volatile collection and analysis of floral scents}

Floral scents emitted from the five Buddleja species were collected in the field using dynamic headspace adsorption techniques during peak flowering times from January to August in 2011 and 2012. Intact inflorescences were carefully enclosed in a modified vacuum dryer, and each sample contained around eight inflorescences from the same plant, with one to five replicates per each population. The inflorescence peduncles were covered with absorbent cotton soaked in a $10 \%$ sucrose solution. The scent-containing air was sucked through glass cartridges containing adsorbent Porapak Q (150 mg, mesh 60/80; Waters Associates, Milford, MA, USA) for 3-5 h (depending on relative strength of the scent to the human nose) during daytime using a pump with an outlet flow rate of $350 \mathrm{ml} \cdot \mathrm{min}^{-1}$. The ambient air pumped into the vacuum dryer was purified with activated carbon. To identify background contamination, ambient air (purified air) was collected as control.

Cartridges were conditioned before sampling by washing with $400 \mathrm{ml}$ dichloromethane, $n$-hexane and acetone for $4 \mathrm{~h}$. The adsorbed scent components were eluted with $c a .0 .8 \mathrm{ml}$ dichloromethane and collected in a $1.5 \mathrm{ml}$ Agilent vial. In each sampling, 1440-72,000 ng n-nonane were added as internal standard (IS) for quantification. The vials were kept in a freezer $\left(-20{ }^{\circ} \mathrm{C}\right)$ until analysis. After odour collection, the total numbers of open flowers in each sample was counted.

The extracts were analysed using coupled gas chromatography and mass spectrometry (GC-MS). Samples were analysed using an Agilent HP 6890 gas chromatograph (Agilent 
Technologies, Santa Clara, CA, USA) equipped with a HP-5MS column $(30 \mathrm{~m} \times 0.25 \mathrm{~mm}, 0.25 \mu \mathrm{m}$ film thickness $)$, linked to a HP5973 mass spectrometer. Helium (He) was used as carrier gas at a flow of $1 \mathrm{ml} \cdot \mathrm{min}^{-1}$, and the injector temperature was set to $250{ }^{\circ} \mathrm{C}$. The column temperature was first set at $40^{\circ} \mathrm{C}$ and then programmed to $250^{\circ} \mathrm{C}$ at a rate of $3{ }^{\circ} \mathrm{C} \cdot \mathrm{min}^{-1}$. Compounds were identified by comparing their retention times (RT) and mass spectra with those of authentic compounds, or by comparison with MS spectra from the Wiley $7 \mathrm{n} .1$ mass spectral library and the associated retention indices reported both in the NIST Chemistry Web Book (http://webbook.nist. gov) and in the RI database (Adams 2001).

Kovats retention indices were calculated using the formula:

$$
\mathrm{I}_{\mathrm{x}}=100_{\mathrm{n}}+100 \times\left(\mathrm{t}_{\mathrm{x}}-\mathrm{t}_{\mathrm{n}}\right) /\left(\mathrm{t}_{\mathrm{n}+1}-\mathrm{t}_{\mathrm{n}}\right)
$$

where $I_{X}$ is the retention index of the compound of interest, $t_{x}$ is the retention time of the compound of interest, $t_{n}$ and $t_{n+1}$ are the retention times of the $n$-alkanes (Sigma-Aldrich Co., St. Louis, MI, USA) eluted immediately before and after the compound of interest, and $n$ is the number of carbon atoms in the $n$-alkane eluted immediately before the compound of interest (Van den Dool \& Dec Kratz 1963; Chen et al. 2012; Da Cunha et al. 2013).

Proportional abundance of compounds (relative amounts with respect to aggregate peak areas, excluding contaminants) of the floral scents was calculated based on the absolute amounts of compounds.

\section{Floral morphology and reward statistical analysis}

To perform multivariate analyses of floral traits (excluding floral scents), number of dimensions for a principal components analysis (PCA) by cross-validation with the MISsMDA package (version 1.7; Husson \& Josse 2013) in R (R Development Core Team 2013) was first estimated. This prerequisite allowed a PCA on the full matrix (898 rows and 11 floral traits measured) with the VEGAN package (version 2.0-5; Oksanen et al. 2012) and the FACTOMineR package (version 1.24; Husson et al. 2013) in $\mathrm{R}$ to be performed.

\section{Floral scent statistical analysis}

Because our 'individuals $\times$ compounds' matrix of floral scents did not meet the assumptions of multivariate normality of variances (Shapiro-Wilk normality test, $\mathrm{W}=0.0908, P<2.2 \mathrm{e}$ 16 , test performed with the MVNORMTEST package (Jarek 2012) in $\mathrm{R}$ ), and of multivariate homogeneity of group dispersions (variances, ANOvA $F_{4,47}=19.494, P=1.636 \mathrm{e}-09$, test performed with the VEGAN package (version 2.0-5; Oksanen et al. 2012) in $\mathrm{R})$, a non-parametric tests to characterise the floral scent differences among samples and Buddleja species was used.

Hellinger transformation, a relativisation by row (sample unit) totals followed by taking the square root of each element in the matrix, to make the floral scent data containing many zeros (e.g., compounds completely absent in certain species, but present in others) suitable for multivariate analysis (Legendre \& Legendre 1998; Legendre \& Gallagher 2001). A non-parametric multiple response permutation procedure (MRPP) with the average Bray-Curtis distances among samples weighted to group size and 999 random permutations (Mielke \& Berry
2001; McCune et al. 2002) was first conducted to test the null hypothesis of no difference in floral scent (relative amounts, in $\%$ ) among species. The MRPP test was performed with the VEGAN package (version 2.0-5; Oksanen et al. 2012) in R. An analysis of similarities (ANOSIM) using the average Bray-Curtis distances among samples and 1000 permutations with the VEGAN package (version 2.0-5; Oksanen et al. 2012) in $\mathrm{R}$ was also conducted as an alternative way to statistically test whether there is a significant difference in floral scent composition among Buddleja species.

To detect floral scent compounds whose presence is statistically associated with certain species, an indicator compound analysis (ICA) with 999 random permutations was performed. The computed indicator value of each compound reflects both its relative abundance (specificity - 'A', the probability that the odour compound belongs to the target species) and its relative frequency (fidelity - 'B', the probability of finding the odour compound in other species). The associated $P$-values determine whether specific compounds are significant indicators of certain species (Dufrêne \& Legendre 1997; De Caceres \& Legendre 2009). The ICA was performed with the INDICSPECIES package (De Caceres \& Legendre 2009) in R.

To characterise the floral scent dissimilarities among samples associated with different groups (e.g., species), a non-metric multi-dimensional scaling (nMDS) ordination based on a matrix of Bray-Curtis dissimilarities, calculated on the relative proportions of odour compounds (in \% of the total blend) was performed. The appropriateness of the nMDS results was determined by comparing, in a Shepard diagram, the distances among samples in the ordination plot with the original distances, and the stress value generated with the nMDS analysis reflects how well the ordination summarises the observed distances among the samples. The nMDS analysis was performed with the VEGAN package (version 2.0-5; Oksanen et al. 2012) in $\mathrm{R}$.

Finally, the contribution of primary scent compounds within species was investigated using PCA in PAST (version 2.08; Hammer et al. 2001). A variance-covariance matrix of the floral scents (relative amount) was used, and the Jolliffe cut-off value obtained provided an informal indication of how many principal components should be considered significant (Jolliffe 1986). Components with eigenvalues smaller than the Jolliffe cut-off can be considered insignificant. The coefficient of each principal component implies the contributions of each compound among populations within a species, and the positive value and negative value of coefficients suggest that they contributed to different populations. The absolute values of coefficients show their contributions.

\section{Breeding systems}

To evaluate the breeding system of the five Buddleja species, inflorescences with floral buds were marked randomly and isolated in parchment paper $(30 \times 25 \mathrm{~cm})$. Five pollination treatments were assigned at peak flowering time: (i) open pollination: inflorescences with floral buds were marked at random and maintained under natural conditions; (ii) geitonogamy: pollen from the same plant individuals was transferred to the stigma of emasculated flowers, and the inflorescences were bagged; (iii) xenogamy: pollen from different individuals (10 $\mathrm{m}$ apart) was transferred to the stigma of emasculated 
flowers, and the inflorescences were bagged; (iv) apomixis: unopened flowers were emasculated and bagged; and (v) pollinator exclusion: the inflorescences were bagged without any manipulations.

Each treatment was conducted on 14 inflorescences from seven plants, and three to five flowers were randomly chosen per inflorescence while other flowers were removed. One population was used to test the breeding system for each species. The marked infructescences of each Buddleja species were harvested and collected around 3 months after the inflorescences were treated. Seed set was calculated as the ratio of expanded seeds to ovules per flower.

\section{RESULTS}

\section{Pollination systems}

Over the duration of the flowering season, a wide spectrum of pollinators was observed foraging on the five Buddleja species throughout the day. Overall, seven different functional groups of pollinator were recorded among these five species: honeybee, bumblebee, butterfly, hawkmoth, hoverfly, fly and sunbird (Fig. 2). No floral visitors were recorded during the nocturnal observation periods.

All five Buddleja species attracted abundant pollinator visits. The evaluation of plant pollination system based on their relative proportions suggested that honeybees and/or
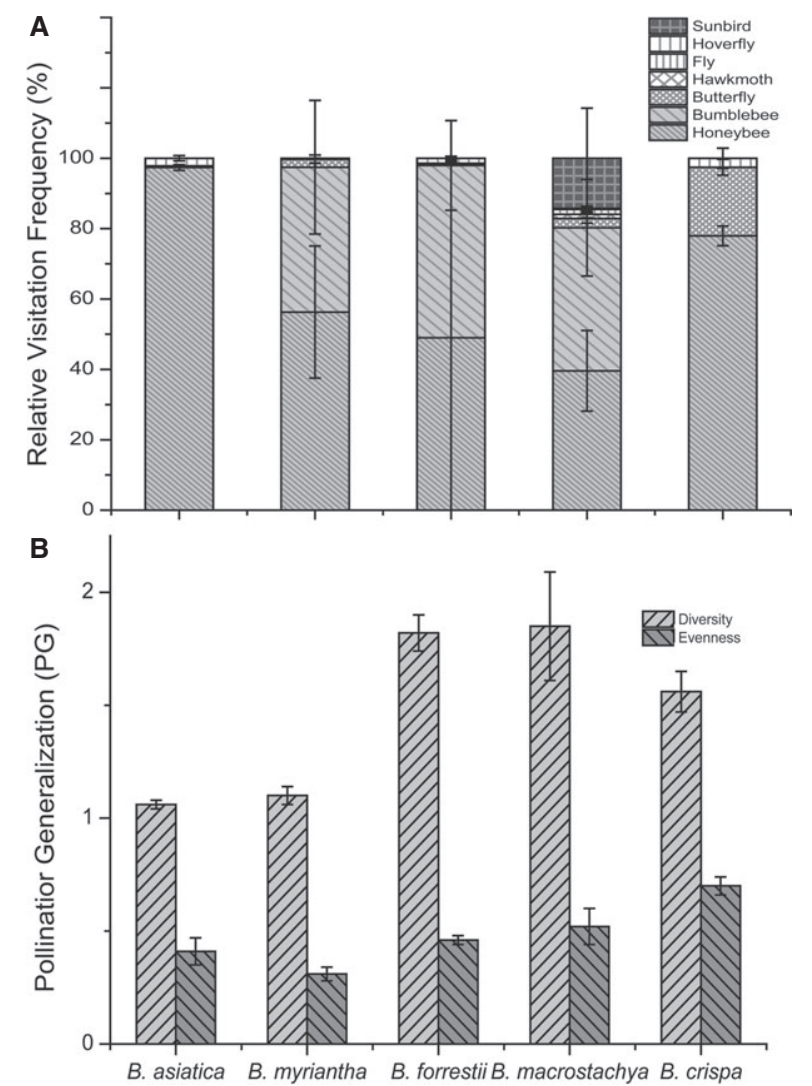

Fig. 2. (A) Relative visitation frequency and (B) pollinator generalisation level of five Buddleja species during 2011 and 2012. Relative visitation frequency and pollination generalisation level are means \pm SE. bumblebees were the major pollinator assemblages. Honeybees were the exclusive pollinator of Buddleja asiatica, constituting $97.38 \pm 0.81 \%$ of the total visits, and other pollinator groups rarely pollinated this species (Fig. 2A). Honeybees were also a major pollinator type for B. crispa $(77.95 \pm 2.79 \%)$, B. forrestii $(49.01 \pm 49.01 \%), \quad$ B. macrostachya $(39.59 \pm 11.46 \%)$ and B. myriantha $(56.30 \pm 18.78 \%)$. In this study, honeybees were mainly Apis cerana, which is native to Asia. Bumblebees were also a major pollinator type for B. forrestii (48.95 $\pm 12.74 \%)$, B. macrostachya $\quad(40.68 \pm 11.46 \%) \quad$ and $\quad$ B. myriantha $(41.16 \pm 18.98 \%)$, but bumblebees never visited B. asiatica and B. crispa during the 2-year field observations (Fig. 2A). Overall, bees (Apis cerana and bumblebee) were the principal insect pollinators for these five Buddleja species, and the total visitation frequency ranged from $77.95 \%$ to $97.9 \%$ (Fig. 2A). Although Buddleja species are commonly known as 'butterfly bush', in these five species, only B. crispa attracted relatively abundant butterflies, accounting for $19.50 \pm 2.25 \%$ of the total visits (Fig. 2A); Papilio xuthus and Pieris rapae were the primary butterfly species. In addition to insect pollinators, a sunbird (Aethopyga nipalensis) was a pollinator of B. macrostachya, accounting for $14.23 \pm 14.23 \%$ of total visits.

With regard to the ecological generalisation levels of these five species, our results showed that pollinator diversity or pollinator generalisation level (PG) was low, ranging from 1.06 to 1.85 , based on pollinator functional groups (Fig. 2B). Although a relatively high PG in $B$. forrestii $(1.82 \pm 0.08)$ and B. macrostachya $(1.85 \pm 0.24)$, both Apis cerana and bumblebees were their major pollinator functional groups, suggesting that bees were the principal pollinator type for the five Buddleja species. The pollinator evenness of these plant species was near 0.5 (Fig. 2B). The reduced evenness of functional pollinator groups in each species suggests changes in the intensity of use for a subset of pre-existing pollinators (Fenster et al. 2004).

A beetle (Antherophagus ochraceus) was found in some inflorescences of $B$. forrestii and $B$. macrostachya, but was not seen to move between flowers during the observation periods, so the hypothesis of beetle pollination in our studied species was rejected.

\section{Floral morphology and floral reward}

Table 1 contains the floral trait data pertaining to attraction, reward and pollen transfer for the five Buddleja species. All Buddleja species investigated here were also characterised by reverse herkogamy (stigma located below the anthers). The anthers of B. asiatica and B. crispa were in the middle of the corolla tube, while others were at the mouth of the corolla. Most floral traits measured varied, except for the corolla tube width and corolla length, which exhibited lower coefficients of variance (between $0.24-6.67 \%$ and $2.05-17.03 \%$, respectively). The flowers of B. asiatica and B. myriantha were smaller than those of B. crispa, B. forrestii and B. macrostachya. All five species had narrow and long corolla tubes, ranging between 1.303.40-mm wide and 5.09-10.59-mm long, respectively (Table 1). All five species produced nectar, which accumulated at the base of the corolla tube, with distinct volumes and concentrations (Table 1).

Our analysis, based on 11 floral morphology and nectar characteristics, showed that a species like $B$. asiatica was more 


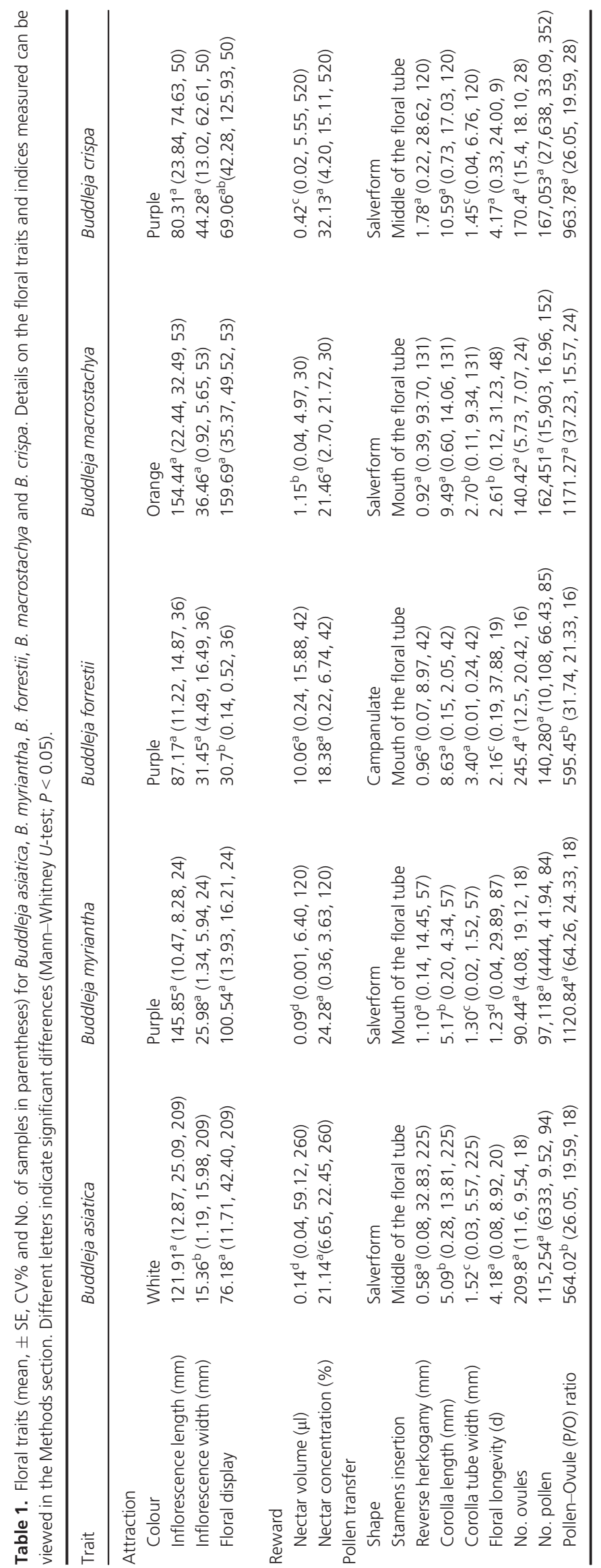


differentiated along the $x$-axis (Fig. S1B), since it was characterised by significantly longer floral longevity and a significantly higher number of ovules compared to the other Buddleja species studied (Table 1). However, floral traits did not exhibit a high degree of clustering according to the Buddleja species investigated (Fig. S1A), suggesting that the floral traits measured cannot reliably be used as diagnostic characters to distinguish each species.

\section{Floral scents}

We identified 78 volatile compounds from the odour samples of the five Buddleja species: fatty acid derivatives (22), benzenoids (8), monoterpenoids (33) and sequiterpenoids (15) (Table S2). Each species emitted distinct volatile compounds, and multiple response permutation procedure (MRPP) analysis indicated that floral scents (relative amounts, in \%) were significantly differentiated among species (MRPP, A $=0.4611$, $\left.\delta_{\text {obs }}=0.3767, \delta_{\text {exp }}=0.699, P<0.01\right)$. The ANOSIM analysis was in accordance with results of the MRPP analysis, which showed a significant difference among Buddleja species (ANOSIM, R $=0.8616, P<0.01)$.

The non-metric multidimensional scaling (nMDS) ordination produced high linear and non-metric fits $\left(\mathrm{R}^{2}=0.895\right.$ and 0.978 , respectively) and a low stress values (0.149; Fig. 3A), illustrating interspecific differentiation in floral scent among
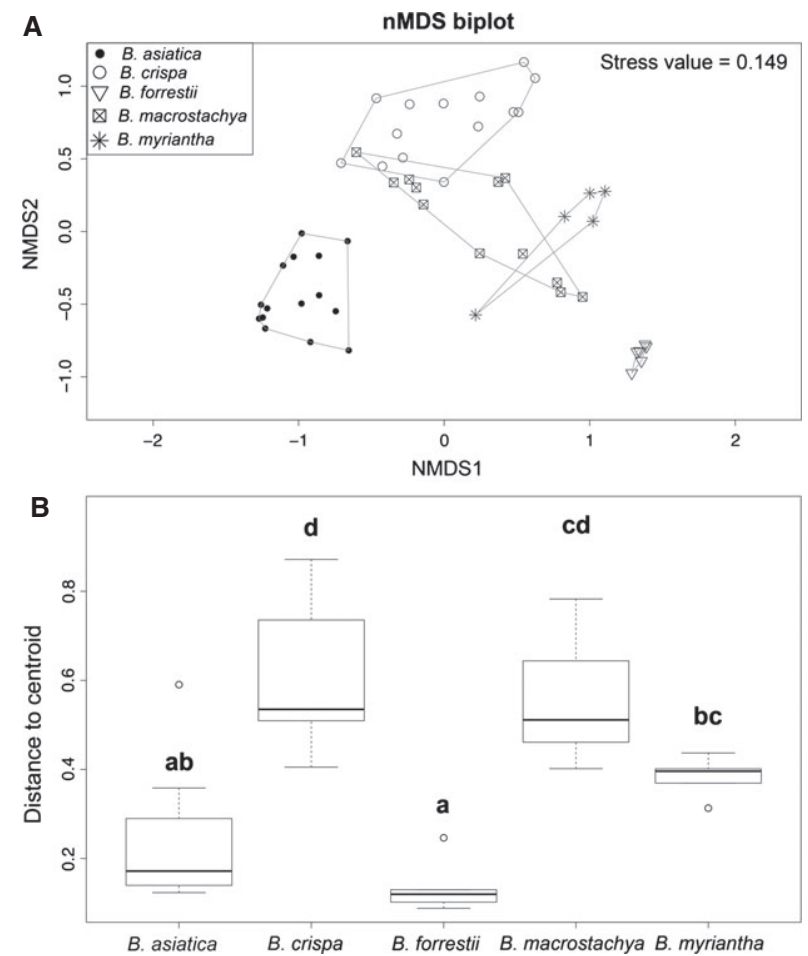

Fig. 3. Floral scent differentiation among the five Buddleja species investigated in the Sino-Himalayan region. A: nMDS biplot of floral scent differentiation based on a matrix of Bray-Curtis dissimilarities calculated on the relative proportions of odour compounds (in\% of total blend). B: Boxplot of intraspecific levels of floral scent dispersion using Bray-Curtis distances among samples (different letters on top of boxplots indicate significant differences with $\alpha=0.05$, tested with Tukey HSD). species. The nMDS biplot indicated that the Buddleja species form discrete clusters (Fig. 3A), except for clusters between B. crispa, B. macrostachya and B. myriantha, which showed a partial overlap. The overlap suggested that $B$. macrostachya has relative higher composition similarity with $B$. crispa and $B$. myriantha. The analysis of intraspecific levels of variance dispersion with Bray-Curtis distances among floral scent samples showed that there were significant differences within species (ANOVA $F_{4,47}=28.994, P=3.615 \mathrm{e}-12$ ). This indicated that $B$. crispa and $B$. macrostachya have the highest intraspecific dissimilarity, B. myriantha was secondary, but B. asiatica and $B$. forrestii have the lowest intraspecific dissimilarity (Fig. 3B). Moreover, significant interspecific differences in the variance dispersion of floral scents are shown in Fig. 3B.

Indicator compound analysis (ICA) detected a significant association between 38 odour compounds within the blends of floral scents and the study species. The results compiled in Table S3 show that, within this group of 38 diagnostic compounds, some are strongly and significantly associated with certain species and can therefore be considered as good 'indicator' or 'predictor' compounds. For example, cis-linalool oxide is a dominant and good 'indicator' volatile compound in the floral scent of B. asiatica; the same applies to cis- $\beta$-ocimene for B. myriantha, $\gamma$-terpinene for $B$. forrestii, $\beta$-cyclocitral for B. macrostachya, and 3-methyl-1-butanol for B. crispa (Table S3). Additionally, strong specificity in floral scent was found in B. forrestii $(13 / 26)$ and B. myriantha (13/29), while more shared volatile compounds were detected in $B$. asiatica (35/37), B. crispa (29/33) and B. macrostachya (35/41) (Table S3).

Finally, the intraspecific contribution of each floral scent compound based on relative amount was evaluated through PCA. PCA1 explained most of the total variance, ranging from $62.35 \%$ to $88.40 \%$ (Fig. S2). Therein, linalool exclusively contributed highly to B. asiatica, and the absolute loading accounted for 0.90 , while most of the floral scent compounds within $B$. asiatica had small contributions, with low relative amounts; cis- $\beta$-ocimene exclusively contributed most to $B$. myriantha $(-0.91)$. Limonene $(-0.86)$ and eucalyptol $(0.45)$ had the highest loading for $B$. forrestii; benzaldehyde (0.75) and lilac aldehyde $(-0.57)$ on B. crispa; and benzaldehyde (0.43), lilac aldehyde $(0.42)$ and limonene $(-0.63)$ on $B$. macrostachya (Fig. S2).

\section{Breeding system}

The mean percentage seed set in control treatments was $68.92 \pm 2.34 \%$ for B. asiatica, $58.53 \pm 2.1 \%$ for B. crispa, $77.60 \pm 2.02 \%$ for B. forrestii, $69.85 \pm 3.05 \%$ for B. macrostachya and $86.63 \pm 2.21 \%$ for B. myriantha (Fig. 4). There was no seed production after geitonogamy treatments in $B$. asiatica and $B$. forrestii. Seed set in pollinator exclusion treatments was significantly disparate among the five species, only $B$. crispa and $B$. macrostachya formed seeds, indicating the occurrence of autogamy. Furthermore, the seed set in these two species was significantly lower than in any other treatments. Thus, all five species require pollinators for increased seed production. In addition, the pollen-ovule (P/O) ratios in the five Buddleja species ranged from $564 \pm 26.05$ to $1171 \pm 37.23$, and were significantly lower in B. asiatica and B. forrestii than in the other three species (Table 1). 


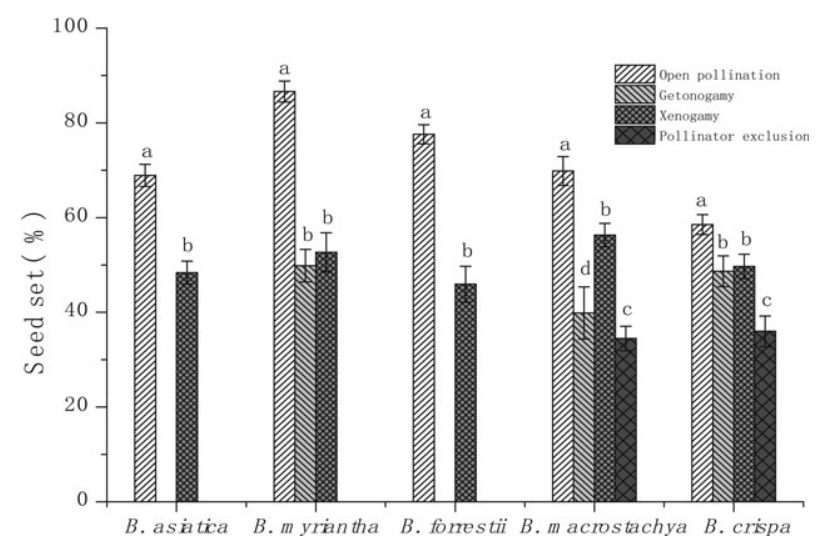

Fig. 4. Seed set per flower under different pollination treatments in five Buddleja species in 2011. Bars are mean $\pm \mathrm{SE}$; different letters above bars indicate significant difference (Mann-Whitney $\boldsymbol{U}$-test; $\boldsymbol{P}<0.05$ ).

\section{DISCUSSION}

Although hand-pollination showed that B. crispa, B. macrostachya and B. myriantha are self-compatible, and B. asiatica and $B$. forrestii are self-incompatible, pollinators contributed greatly to their reproduction, as significant differences in seed set were observed between pollinator exclusion and control treatments (Fig. 4). This suggested that all five Buddleja species are actually pollinator-dependent. Bees are the principal pollinators (Fig. 2), and all five species are specialised for bee pollination (i.e., at least $75 \%$ of the time by a single functional group; PG value near 1), based on the evaluated specialisation in plant pollination system (Fenster et al. 2004; Sahli \& Conner 2006). Honeybees (Apis cerana) and/or bumblebees were the major bee pollinators (Fig. 2). Buddleja asiatica and B. crispa were nearly exclusively pollinated by Apis cerana, while the high-altitude species, B. forrestii, B. macrostachya and B. myriantha, were also pollinated by bumblebees (Fig. 2).

All five Buddleja species had narrow and long corolla tubes, with concealed nectar (Table 1). According to the typology of (Ellis \& Ellis-Adam 1993; Waser 2006), larger bees and Lepidoptera should be major insect pollinators for the five Buddleja species, despite Buddleja being known as attractive to butterflies (Faegri \& Van der Pijl 1979; Proctor et al. 1996; Norman 2000). Field observations indicated that bees were the primary pollinators of all five Asian Buddleja species studied, rather than butterflies. Only B. crispa attracted abundant butterflies (19.50 $\pm 2.25 \%$ ). In contrast, $B$. davidii shares similar morphological traits with the five studied Buddleja species, yet is mainly butterfly- and moth-pollinated (Anderson 2003; Guédot et al. 2008; Chen et al. 2011), but not bee-pollinated. Therefore, the interspecific differences in primary pollination systems in Buddleja cannot be interpreted with the Ellis \& Ellis-Adam typology alone. Actually, bee and butterfly pollination syndromes show much overlap, and are difficult to distinguish unless they are effectively diagnostic syndromes (Ollerton \& Watts 2000). In this study, we analysed 11 different morphology and nectar characteristics, and found that none of them served as diagnostic characteristics to further distinguish between bee and butterfly pollination syndromes among the five studied species (Fig. S1).

Floral scent constitutes an ancient and important channel of communication between flowering plants and their pollinators
(Pellmyr \& Thien 1986; Raguso 2008; Schiestl \& Dötterl 2012), and plays important roles in plant-pollinator interactions in angiosperms (Pellmyr \& Thien 1986; Svensson et al. 2005; Smith 2010). In scented flowers, floral scent composition is closely associated with their dominant pollinator assemblage, and potential pollinator types can be predicted based on floral scent compounds (Schiestl et al. 2003; Brodmann et al. 2008; Shuttleworth \& Johnson 2009), including in B. davidii (Andersson et al. 2002; Andersson \& Dobson 2003; Guédot et al. 2008). In the present study, monoterpenoids $(42.66 \pm 10.79 \%-95.59 \pm 1.50 \%$, in terms of relative amount) were the predominant floral scents in the five studied species, especially monoterpene alkenes, such as limonene, cis- $\beta$-ocimene and trans- $\beta$-ocimene (Table S2). This suggests that butterflies might not be their major pollinator because butterflypollinated plants do not emit large amounts of monoterpene alkenes (Andersson et al. 2002; Andersson \& Dobson 2003). B. crispa also emitted more benzenoids ( $45.34 \pm 10.17 \%)$, which could be attractive to a variety of butterflies (Honda et al. 1998; Andersson \& Dobson 2003; Guédot et al. 2008), as well as $42.66 \pm 10.79 \%$ of monoterpenoids (Table S2), and this species was indeed the only one that attracted a relatively large proportion of butterflies (Fig. 2).

Floral scent is a complex phenotypic trait, with diverse chemical compositions and relative amounts of compounds, and each factor may play a role in floral scent-pollinator interactions. Therein, species-specific volatile compositions can guide food-searching insects to facilitate locating a specific rewarding species or avoid non-rewarding species (Andersson \& Dobson 2003; Borg-Karlson et al. 2003; Chen et al. 2009; Schiestl \& Dötterl 2012). Our results suggest the occurrence of species-specific floral scent compounds among the five Buddleja species, because their floral scents showed a tendency to diverge (Fig. 3). We detected the species-specific scent compounds, or 'indicator' compounds, of each species with high intraspecific specificity and fidelity (Table S3). Interestingly, most 'indicator' compounds detected from the Buddleja species can elicit strong responses in the antennae of bees in other angiosperms, e.g., cis-linalool oxide, 3-methyl-1-butanol, $\alpha$ caryophyllene, 4,8-dimethylnona-1,3,7-triene, terpinolene, $\beta$ pinene, trans- $\beta$-bergamotene, tetradecane and cis- $\beta$-ocimene (Dani et al. 1998; Rose et al. 1998; Ônsoli et al. 2002; Fortunato et al. 2004; Ono 2005; Chen \& Song 2008; Rohrig et al. 2008), and hence can serve as attraction signals to bees. This suggests that bees were a potential pollinator type for the five Buddleja species. In general, for bee-pollinated flowers, a single compound of larger relative amount has been shown to have a food signalling potential as high as that of the whole scent blend (Borg-Karlson et al. 2003; Chen et al. 2009). However, all the detected 'indicator' compounds within the species emitted low relative quantities; only cis- $\beta$-ocimene in B. myriantha had a larger relative amount $(19.72 \pm 7.84 \%$; Table S2).

Although the roles of floral scent compounds in chemical communication with pollinators have been reported in different flowers, the importance of relative amount of scent compounds has rarely been highlighted (Jürgens et al. 2002; Andersson \& Dobson 2003; Borg-Karlson et al. 2003; Parachnowitsch et al. 2012). In the present study, the contribution of floral scent compounds within species was assessed based on relative amount, and six different compounds with the largest intraspecific contribution were detected from these five species: 
linalool, cis- $\beta$-ocimene, limonene, eucalyptol, benzaldehyde and lilac aldehyde (Fig. S2). Associated with our field observations, we found that bee-pollinated B. asiatica, B. forrestii, $B$. macrostachya and $B$. myriantha emitted larger relative amounts of linalool, limonene and eucalyptol, limonene and cis- $\beta$-ocimene than other volatile compounds (Fig. S2). Interestingly, all five scent compounds have been shown to stimulate foraging behaviour in bees (Dani et al. 1998; Borg-Karlson et al. 2003; Eltz et al. 2006; Rohrig et al. 2008; Jarau et al. 2012). Among the five Buddleja species, only B. crispa emitted abundant benzaldehyde and lilac aldehyde, which can elicit strong foraging behaviour in butterflies (Honda 1980; Schulz et al. 1993; Honda et al. 1998; Anderson 2003; Andersson \& Dobson 2003; Dötterl et al. 2006; Guédot et al. 2008). This is consistent with our field observations that butterflies also play important roles in B. crispa pollination, but not in the other four Buddleja species (Fig. 2). This provides strong evidence that the attraction of butterflies in B. crispa is associated with the larger emission of benzaldehyde and lilac aldehyde. In addition, butterfly-pollinated $B$. davidii also emits larger relative amounts of butterfly attractants, such as 4-oxoisophorone and benzaldehyde (Andersson \& Dobson 2003; Guédot et al. 2008; Chen et al. 2011). Overall, floral scent compounds with largest relative amounts within a species were also closely associated with their primary pollinator assemblage. This supports the hypothesis of Parachnowitsch et al. (2012), that larger emission of a specific floral scent compound in these Buddleja species could be associated with specific pollinator preferences of principal pollinators.

Generally, the typology of Ellis \& Ellis-Adam can be used to interpret plant-pollinator interactions in Buddleja species. However, this approach is not effective for all Buddleja species because it cannot interpret interspecific pollinator differences. In contrast, floral scent composition, especially species-specific scent compounds and principal compounds with larger relative amounts are closely associated with their principal pollinator assemblage. Bees (honeybee/bumblebee) are the principal pollinators; therefore, we conclude that it is floral scent composition rather than morphology traits that should be used to interpret plant-pollinator interactions among the five studied Buddleja species.

\section{ACKNOWLEDGEMENTS}

We thank J.-H. Ding and D.-H. Yang for valuable help with fieldwork. We particularly thank Dr. C.-Q. Liu, Z.-Q. Zhang, S.-X. Chen, S.-L. Luo and A,-R. Li for advice on the manuscript. This study was supported by grants-in-aid from the National Natural Science Foundation of China (Grant no. 30970192 to W.-B. Sun; 31100177 to G. Chen). N. J. Vereecken was financially supported by the Belgian FRS-FNRS through a post-doctoral grant (Chargé de Recherches, 2008-2012).

\section{SUPPORTING INFORMATION}

Additional Supporting Information may be found in the online version of this article:

Figure S1. Principal components analysis (PCA) using floral morphology data from five Buddleja species investigated in the Sino-Himalayan region.

Figure S2. Principal components analysis (PCA) 1 loadings of floral scents contribution among individuals within a species are displayed. PCA1 explained variance is listed in parentheses. The loading coefficients are based on var-covar matrix of relative amounts of floral scent composition.

Table S1. GPS coordinates and sample sizes (number of plants) of five Buddleja species used in this study. The abbreviations of populations for pollination observation are in bold.

Table S2. Floral scent compounds of five Buddleja species investigated. Mean $\pm \mathrm{SE}$, relative amounts (in\%) of odour compounds in headspace fractions of B. asiatica, B. myriantha, B. forrestii, B. macrostachya and B. crispa ( $t r$ indicates trace amounts, i.e., compounds with concentration $<0.1 \%$ ). The compounds are ordered in classes, and listed according to relative retention time (KRI) within each class. Chemical Abstracts Service (CAS) registry numbers and literature relative retention time (KRI) are also provided. Different letters indicate significant differences (Mann-Whitney $U$-test; $P(0.05)$.

Table S3. Indicator compound analysis (ICA) performed on Buddleja floral scents to identify individual compounds significantly associated with a single Buddleja species. The statistics are provided along with associated $P, A$ (specificity) and $\mathrm{B}$ (fidelity) values in parentheses (see Methods for details).

\section{REFERENCES}

Adams R. (2001) Identification of essential oil components by gas chromatography /quadrupole mass spectroscopy. Allured Publishing Corp., Chicago, IL, USA.

Anderson S. (2003) Antennal responses to floral scents in the butterflies Inachis io, Aglais urticae (Nymphalidae), and Gonepteryx rhamni (Pieridae). Chemoecology, 12, 13-20.

Andersson S., Dobson H.M. (2003) Antennal responses to floral scents in the butterfly Heliconius melpomene. Journal of Chemical Ecology, 29, 2319-2330.

Andersson S., Nilsson L.A., Groth I., Bergström G. (2002) Floral scents in butterfly-pollinated plants: possible convergence in chemical composition. Botanical Journal of the Linnean Society, 140, 129-153. Borg-Karlson A.K., Tengo J., Valterova I., Unelius C.R., Taghizadeh T., Tolasch T., Francke W. (2003) (S)-(+)-linalool, a mate attractant pheromone component in the bee Colletes cunicularius. Journal of Chemical Ecology, 29, 1-14.
Brodmann J., Twele R., Francke W., Hölzler G., Zhang Q.H., Ayasse M. (2008) Orchids mimic green-leaf volatiles to attract prey-hunting wasps for pollination. Current Biology, 18, 740-744.

Chen C., Song Q. (2008) Responses of the pollinating wasp Ceratosolen solmsi marchali to odor variation between two floral stages of Ficus hispida. Journal of Chemical Ecology, 34, 1536-1544.

Chen G., Sun W.B., Sun H. (2007) Ploidy variation in Buddleja L. (Buddlejaceae) in the Sino-Himalayan region and its biogeographical implications. Botanical Journal of the Linnean Society, 154, 305312 .

Chen C., Song Q., Proffit M., Bessière J.M., Li Z., Hossaert-McKey M. (2009) Private channel: a single unusual compound assures specific pollinator attraction in Ficus semicordata. Functional Ecology, 23, 941-950.

Chen G., Sun H., Sun W., Norman E. (2011) Buddleja davidii and Buddleja yunnanensis: exploring features associated with commonness and rarity in Buddleja.
Flora - Morphology. Distribution, Functional Ecology of Plants, 206, 892-895.

Chen G., Gong W.C., Ge J., Dunn B.L., Sun W.B. (2012) Floral scents of typical Buddleja species with different pollination syndromes. Biochemical Systematics and Ecology, 44, 173-178.

Da Cunha M., Schneider J.K., Brasil M.C., Cardoso C.A., Monteiro L.R., Mendes F.L., Pinho A., Jacques R.A., Machado M.E., Freitas L.S., Caramão E.B. (2013) Analysis of fractions and bio-oil of sugar cane straw by one-dimensional and two-dimensional gas chromatography with quadrupole mass spectrometry (GC $\times \mathrm{GC} / \mathrm{qMS})$. Microchemical Journal, 110, $113-119$.

Dani F.R., Morgan E.D., Jones G.R., Turillazzi S., Cervo R., Wittko F. (1998) Species-specific volatile substances in the venom sac of hover wasps. Journal of Chemical Ecology, 24, 1091-1104.

Darwin C. (1862) On the various contrivances by which British and foreign orchids are fertilized. John Murray, London, UK: 365 pp. 
De Caceres M.D., Legendre P. (2009) Associations between species and groups of sites: indices and statistical inference. Ecology, 90, 3566-3574.

Delpino F. (1873-1874) Ulteriori osservazioni e considerazioni sulla dicogamia nel regno vegetale. Atti della Società Italiana di Scienze Naturali, 16, 151-349, 17, 266-407.

Dötterl S., Burkhardt D., Weißbecker B., Jürgens A., Schütz S., Mosandl A. (2006) Linalool and lilac aldehyde/alcohol in flower scents: Electrophysiological detection of lilac aldehyde stereoisomers by a moth. Journal of Chromatography A, 1113, 231-238.

Dufrêne M., Legendre P. (1997) Species assemblages and indicator species: the need for a flexible asymmetrical approach. Ecological Monographs, 67, 345366.

Ellis W.N., Ellis-Adam A.C. (1993) To make a meadow it takes a clover and a bee: the entomophilous flora of N.W. Europe and its insects. Bijdragen tot de Dierkunde, 63, 193-220.

Eltz T., Ayasse M., Lunau K. (2006) Species-specific antennal responses to tibial fragrances by male orchid bees. Journal of Chemical Ecology, 32, 71-79.

Endress P.K. (1996) Diversity and evolutionary biology of tropical flowers. Cambridge University Press, Cambridge, UK.

Faegri K., Van der Pijl L. (1979) The principles of pollination ecology. Pergamon Press, Oxford, UK.

Fenster C.B., Armbruster W.S., Wilson P., Dudash M.R., Thomson J.D. (2004) Pollination syndromes and floral specialization. Annual Review of Ecology Evolution and Systematics, 35, 375-403.

Fortunato A., Dani F.R., Sledge M.F., Fondelli L., Turillazzi S. (2004) Alarm communication in Ropalidia social wasps. Insectes Sociaux, 51, 299-305.

Galen C. (1989) Measuring pollinator-mediated selection on morphometric floral traits: bumblebees and the alpine sky pilot, Polemonium viscosum. Evolution, 88, 2-890.

Gong Y.B., Huang S.Q. (2009) Floral symmetry: pollinator-mediated stabilizing selection on flower size in bilateral species. Proceedings of the Royal Society B: Biological Sciences, 276, 4013-4020.

Gong Y.B., Huang S.Q. (2011) Temporal stability of pollinator preference in an alpine plant community and its implications for the evolution of floral traits. Oecologia, 166, 671-680.

Guédot C., Landolt P.J., Smithhisler C.L. (2008) Odorants of the flowers of butterfly bush, Buddleja davidii, as possible attractants of pest species of moths. Florida Entomologist, 91, 576-582.

Hammer Ø., Harper D.A.T., Ryan P.D. (2001) PAST: paleontological statistics software package for education and data analysis. Palaeontologia Electronica, 4, 83-93.

Honda K. (1980) Odor of a papilionid butterfly. Odoriferous substances emitted by Atrophaneura alcinous alcinous (Lepidoptera: Papilionidae). Journal of Chemical Ecology, 6, 867-873.

Honda K., Ômura H., Hayashi N. (1998) Identification of floral volatiles from Ligustrum japonicum that stimulate flower-visiting by cabbage butterfly, Pieris rapae. Journal of Chemical Ecology, 24, 2167-2180.

Huang S.Q., Fenster C.B. (2007) Absence of long-proboscid pollinators for long-corolla-tubed Himalayan Pedicularis species: implications for the evolution of corolla length. International Journal of Plant Sciences, 168, 325-331.

Husson F., Josse J. (2013) missMDA: handling missing values with/in multivariate data analysis (principal component methods). R Foundation for Statistical Computing, Vienna, Austria.

Husson F., Josse J., Lê S., Mazet J. (2013) FactoMineR: multivariate exploratory data analysis and data mining with R. R package version 1.25. Available from http://factominer.free.fr (accessed 21 February 2014).

Jarau S., Žácek P., Šobotník J., Vrkoslav V., Hadravová R., Coppée A., Vašícková S., Jiroš P., Valterová I. (2012) Leg tendon glands in male bumblebees (Bombus terrestris): structure, secretion chemistry, and possible functions. Naturwissenschaften, 99, 1039 1049.

Jarek S. (2012) Mvnormtest: normality test for multivariate variables. Available from http://CRAN. R-project.org $/$ package $=$ mvnormtest $/$ (accessed 20 April 2013).

Jolliffe I.T. (1986) Principal component analysis. Springer, New York, NY, USA.

Jürgens A., Witt T., Gottsberger G. (2002) Flower scent composition in night-flowering Silene species (Caryophyllaceae). Biochemical Systematics and Ecology, 30, 383-397.

Kulbaba M.W., Worle A.C. (2012) Selection on floral design in Polemonium brandegeei (Polemoniaceae): female and male fitness under hawkmoth pollination. Evolution, 66, 1344-1359.

Legendre P., Gallagher E.D. (2001) Ecologically meaningful transformations for ordination of species data. Oecologia, 129, 271-280.

Legendre P., Legendre L. (1998) Numerical ecology, 2nd edition. Elsevier, Amsterdam, the Netherlands.

Li P.T., Leeuwenberg A.J.M. (1996) Loganiaceae. In: Wu C. Y., Raven P. H. (Eds), Flora of china, Vol 15. Science Press, Beijing, China, pp 320-332.

Mao Y.Y., Huang S.Q. (2009) Pollen resistance to water in 80 angiosperm species: flower structures protect rain-susceptible pollen. New Phytologist, 183, 892 899.

McCune B., Grace J.B., Urban D.L. (2002) Analysis of ecological communities. MjM Software Design, Gleneden Beach, OR, USA.

Mielke P.W. Jr, Berry K.J. (2001) Permutation methods: a distance function approach. Springer, Berlin, Germany.

Moore R.J. (1947) Cytotaxonomic studies in the Loganiaceae. I. Chromosome numbers and phylogeny in the Loganiaceae. American Journal of Botany, 34, 527-538.

Müller H. (1881) Die alpenblumen, ihre befruchtung durch insecten und ihre anpassungen an dieselben. Engelmann, Leipzig, Germany.

Nie Z.L., Gu Z.J., Sun H. (2005) Polyploidy in the flora of the Hengduan Mountains hotspot, southwestern China. Annals of the Missouri Botanical Garden, 92, 275-306.

Norman E.M. (2000) Buddlejaceae. flora neotropica monograph, 81. The New York Botanical Garden, Bronx, NY, USA, pp 1-190.

Oksanen J., Blanchet F.G., Kindt R., Legendre P., Minchin P.R., O'Hara R.B., Simpson G.L., Solymos P., Stevens M.H.H. (2012) Vegan: community ecology package. $R$ package version 2.0-2. R Foundation for Statistical Computing, Vienna, Austria.

Ollerton J., Watts S. (2000) Phenotype space and floral typology: towards an objective assessment of pollination syndromes. Det Norske Videnskaps-Akademi. I. Matematisk-Naturvidenskapelige Klasse Skrifter, Ny Serie, 39, 149-159.

Ollerton J., Johnson S.D., Hingston A.B. (2006) Geographical variation in diversity and specificity of pol- lination systems. In: Waser N. M., Ollerton J. (Eds), Plant-pollinator interactions: from specialization to generalization. University of Chicago Press, Chicago, IL, USA, pp 283-308.

Ollerton J., Alarcón R., Waser N.M., Price M.V., Watts S., Cranmer L., Hingston A., Peter C.I., Rotenberry J. (2009) A global test of the pollination syndrome hypothesis. Annals of Botany, 103, 1471-1480.

Ono M. (2005) Semiochemicals that regulate social behaviour of hornets. Aroma Research, 6, 230-236.

Ônsoli F.L., Williams H.J., Vinson S.B., Matthews R.W., Cooperband M.F. (2002) Trans-bergamotenes - male pheromone of the ectoparasitoid Melittobia digitata. Journal of Chemical Ecology, 28, 16751689.

Parachnowitsch A.L., Kessler A. (2010) Pollinators exert natural selection on flower size and floral display in Penstemon digitalis. New Phytologist, 188, 393-402.

Parachnowitsch A.L., Raguso R.A., Kessler A. (2012) Phenotypic selection to increase floral scent emission, but not flower size or colour, in bee-pollinated Penstemon digitalis. New Phytologist, 195, 667-675.

Pauw A. (2006) Floral syndromes accurately predict pollination by a specialized oil-collecting bee (Rediviva peringueyi, Melittidae) in a guild of South African orchids (Coryciinae). American Journal of Botany, 93, 917-926.

Pellmyr O., Thien L.B. (1986) Insect reproduction and floral fragrances: keys to the evolution of the angiosperms. Taxon, 35, 76-85.

Proctor M., Yeo P., Lack A. (1996) The natural history of pollination. Harper Collins, London, UK.

Raguso R.A. (2001) Floral scent, olfaction, and scentdriven foraging behavior. In: Chittka L., Thompson J. D. (Eds), Cognitive ecology of pollination. Cambridge University Press, Cambridge, UK, pp 83-105.

R Development Core Team (2013) R: A Language and Environment for Statistical Computing, R Foundation for Statistical Computing, Vienna, Austria.

McCune B., Grace J.B., Urban D.L. (2002) Analysis of ecological communities. MjM Software Design, Gleneden Beach, OR, USA.

Rohrig E., Sivinski J., Teal P., Stuhl C., Aluja M. (2008) A floral-derived compound attractive to the tephritid fruit fly parasitoid Diachasmimorpha longicaudata (Hymenoptera: Braconidae). Journal of Chemical Ecology, 34, 549-557.

Rose U.S.R., Lewis W.J., Tumlinson J.H. (1998) Specificity of systemically released cotton volatiles as attractants for specialist and generalist parasitic wasps. Journal of Chemical Ecology, 24, 303-319.

Sahli H.F., Conner J.K. (2006) Characterizing ecological generalization in plant-pollination systems. Oecologia, 148, 365-372.

Schiestl F.P. (2010) The evolution of floral scent and insect chemical communication. Ecology Letters, 13, 643-656.

Schiestl F.P., Dötterl S. (2012) The evolution of floral scent and olfactory preferences in pollinators: coevolution or pre-existing bias. Evolution, 66, 2042-2055. Schiestl F.P., Peakall R., Mant J.G., Ibarra F., Schulz C., Franke S., Francke W. (2003) The chemistry of sexual deception in an orchid-wasp pollination system. Science, 302, 437-438.

Schulz S., Boppre M., Vane-Wright R.I. (1993) Specific mixtures of secretions from male scent organs of African milkweed butterflies (Danainae). Philosophical Transactions of the Royal Society of London Series B-Biological Sciences, 342, 161-181. 
Shuttleworth A., Johnson S.D. (2009) The importance of scent and nectar filters in a specialized wasp-pollination system. Functional Ecology, 23, 931-940.

Smith S.D.W. (2010) Using phylogenetics to detect pollinator-mediated floral evolution. New Phytologist, 188, 354-363.

Soler C., Proffit M., Chen C., Hossaert-McKey M. (2010) Private channels in plant-pollinator mutualisms. Plant Signaling and Behavior, 5, 893-895.

Svensson G.P., Hickman M.O. Jr, Bartram S., Boland W., Pellmyr O., Raguso R.A. (2005) Chemistry and geographic variation of floral scent in Yucca filamentosa (Agavaceae). American Journal of Botany, 92, 1624-1631.
Van den Dool H., Dec Kratz P.A. (1963) generalization of the retention index system including linear temperature programmed gas-liquid partition chromatography. Journal of Chromatography A, 11, 463-471.

Van der Pijl L. (1961) Ecological aspects of flower evolution. II. Zoophilous flower classes. Evolution, 15, 44-59.

Vogel S. (1954) Blütenbiologische Typenals Elemente der Sippengliederung, dargestellt anhand der Flora Südafrikas. Botanische Studien, 1, 1-338.

Waser N.M., Ollerton J. (Eds) (2006) Plant-pollinator interactions: from specialization to generalization. University of Chicago Press, Chicago, IL, USA.
Waser N.M., Chittka L., Price M.V., Williams N.M., Ollerton J. (1996) Generalization in pollination systems, and why it matters. Ecology, 77, 1043-1060.

Webb C.J., Lloyd D.G. (1986) The avoidance of interference between the presentation of pollen and stigmas in angiosperms II. Herkogamy. New Zealand Journal of Botany, 24, 163-178.

Willmer P. (2011) Pollination and floral ecology. Princeton University Press, Princeton, NJ, USA, pp 322-336.

Wyatt R., Broyles S.B., Lipow S.R. (2000) Pollen-ovule ratios in milkweeds (Asclepiadaceae): an exception that probes the rule. Systematic Botany, 25, 171-180. 